\title{
Twenty-first intercountry meeting for directors of poliovirus laboratories in the WHO Eastern Mediterranean Region ${ }^{1}$
}

Citation: Twenty-first intercountry meeting for directors of poliovirus laboratories in the WHO Eastern Mediterranean Region. East Mediterr Health J. 2020;26(7):864-865 https://doi.org/10.26719/2020.26.7.864

Copyright (C) World Health Organization (WHO) 2020. Open Access. Some rights reserved. This work is available under the CC BY-NC-SA 3.0 IGO license (https://creativecommons.org/licenses/by-nc-sa/3.o/igo)

\section{Introduction}

The twenty-first intercountry meeting for directors of poliovirus laboratories in the World Health Organization (WHO) Eastern Mediterranean Region was held by the WHO Regional Office for the Eastern Mediterranean in Muscat, Oman, on 22-24 January 2020 (1). The meeting was attended by directors of poliovirus laboratories in 12 countries, including: Egypt, Iraq, Islamic Republic of Iran, Jordan, Kuwait, Morocco, Oman, Pakistan, Saudi Arabia, Syrian Arab Republic, Sudan and Tunisia. Participants also included experts from: Centers for Disease Control and Prevention (CDC), United States of America; National Institute for Biological Standards and Control (NIBSC), United Kingdom; National Institute of Public Health and the Environment (RIVM), Netherlands; Kenya Medical Research Institute (KEMRI), Kenya; and the National Virology Laboratory, Zimbabwe. The meeting's secretariat included staff from WHO headquarters, Geneva, Switzerland, and the WHO Regional Office for the Eastern Mediterranean, Cairo, Egypt.

The specific objectives of the meeting were to:

- review the regional polio laboratory network performance;

- provide technical information on issues related to the global polio eradication initiative;

- discuss the role of poliovirus laboratories in the polio end-game strategy, Global Action Plan III (GAPIII) phase 1 activities and environmental surveillance; and

- develop recommendations for further improvement in laboratory performance.

The meeting was attended by over 45 regional and international experts, including officials from sister United Nations (UN) agencies, representatives of humanitarian organizations, development partners and donors, academics from Lebanon, Netherlands, Qatar, United Kingdom and United States of America, as well as senior WHO staff from the Regional Office for the Eastern Mediterranean, Cairo, Egypt, WHO headquarters, Geneva, Switzerland, and country offices in the Region.

\section{Summary of discussions}

WHO global and regional polio laboratory coordinators gave presentations on the current state of the Global Po- lio Eradication Initiative (GPEI) and an overview of the performance, activities and challenges faced by the WHO Global Polio Laboratory Network (GPLN) and planned endgame strategies. They congratulated laboratory personnel on achieving the landmark certification of wild poliovirus type 3 (WPV3) eradication (2) and expressed appreciation for their commitment to eradicating wild poliovirus type 1 . They noted that the increasing number of circulating vaccine-derived poliovirus type 2 (cVDPV2) outbreaks, since the switch from trivalent oral polio vaccine (tOPV) to bivalent oral polio vaccine (bOPV) in 2016, had led to a corresponding increase in GPLN workload.

They highlighted that the ongoing cVDPV2 outbreaks must be controlled by implementing the new strategies that are being developed. The achievements of polio laboratories in the Region, including the efficient and high quality work of laboratories for both acute flaccid paralysis (AFP) and environmental surveillance, and the establishment of new laboratories able to conduct intra-typic differentiation of polioviruses by real time polymerase chain reaction (RT-PCR) in Jordan and Morocco, were commended.

Key aspects of the regional network's laboratory quality assurance programme were discussed, with a focus on biosafety related to the handling of poliovirus. All 16 elements of the biorisk management system described in Annex 6 of the WHO global action plan (GAPIII) (3) will be assessed for all laboratories in the Region. Performance in the Region continues to be excellent, with laboratories providing high quality information on poliovirus isolation and characterization in a timely manner, which is essential for guiding GPEI activities. Laboratories in the Region are also actively engaged in supporting endgame activities such as pilot testing of improved laboratory methods.

\section{Recommendations}

\section{To WHO}

- The Regional Coordinator, in collaboration with WHO headquarters, should work towards establishment of a laboratory surge hub to support laboratories in:

- identifying and overcoming limitations affecting optimal performance, such as in logistics, resources and training; 
- facilitating quick responses to increasing/changing testing demands; and

- facilitating additional training for implementation of new methods and for refreshing purposes.

- collaborating with CDC on training of laboratories' staff on implementation of new direct detection assays; and

- ensuring that laboratories' staff are aware of use of novel OPV2 (nOPV2) strains.

\section{To Member States}

- The heads of laboratories, in collaboration with ministries of health and national authorities/stakeholders, should work with WHO to ensure the use of national resources to maintain the highest level of polio surveillance and respond to the increasing testing demands required for the completion of global polio eradication, including:
- identifying alternative resources for sustaining laboratory functions at certification standards to address the potential reduction in GPEI resources; and

- working with national vaccine preventable disease surveillance and health emergency teams to prioritize integration of existing polio assets in other relevant public health priority areas.

- ensuring that each laboratory completes a comprehensive contingency plan to address the challenges in outbreak or emergency settings placed on testing demand and submit the plan to the Regional Coordinator for review.

- ensuring that laboratory staff contribute to regular monitoring/review of all environmental surveillance sites for sensitivity, functionality and country relevance, using recommended WHO environmental surveillance laboratory performance indicators.

\section{References}

1. World Health Organization Regional Office for the Eastern Mediterranean (WHO/EMRO). Summary Report on the Twenty-first intercountry meeting for directors of poliovirus laboratories in the WHO Eastern Mediterranean Region. Cairo: WHO/EMRO; 2020 (https://applications.emro.who.int/docs/WHOEMPOL441E-eng.pdf?ua=1\&ua=1).

2. World Health Organization. WHO DG: Thank you to all who made WPV3-free world possible. Geneva: World Health Organization; 2019 (http://polioeradication.org/news-post/day-after-wpv3-declared-as-eradicated-who-dg-thanks-all-stakeholders-whomade-this-possible/).

3. World Health Organization. Global action plan to minimize poliovirus facility-associated risk after type-specific eradication of wild polioviruses and sequential cessation of oral polio vaccine use - GAPIII. Geneva: World Health Organization; 2015 (https:// apps.who.int/iris/bitstream/handle/10665/208872/WHO_POLIO_15.05_eng.pdf?sequence=1\&isAllowed=y) 